\title{
IgG4 thyroiditis in the Asian population
}

\author{
Yaqiong $\mathrm{Li}^{1}$, Keiko Inomata ${ }^{2}$, Eijun Nishihara ${ }^{3}$, Kennichi Kakudo ${ }^{4}$ \\ ${ }^{1}$ Department of Pathology, Shandong Provincial Hospital Affiliated to Shandong First Medical University, Jinan, China; ${ }^{2}$ Department of Clinical and \\ Laboratory Medicine, Yamashita Thyroid Hospital, Fukuoka, Japan; ${ }^{3}$ Department of Internal Medicine, Kuma Hospital, Kobe, Japan; ${ }^{4}$ Department \\ of Pathology and Thyroid Disease Center, Izumi City General Hospital, Izumi, Japan \\ Contributions: (I) Conception and design: Y Li; (II) Administrative support: Y Li; (III) Provision of study materials or patients: Y Li, E Nishihara; \\ (IV) Collection and assembly of data: All authors; (V) Data analysis and interpretation: All authors; (VI) Manuscript writing: All authors; (VII) Final \\ approval of manuscript: All authors. \\ Correspondence to: Yaqiong Li, MD, PhD. Department of Pathology, Shandong Provincial Hospital Affiliated to Shandong First Medical University, \\ 324\#, Jingwuweiqi Road, Jinan, China. Email: ranran1007@126.com.
}

\begin{abstract}
Immunoglobulin G4-related disease (IgG4-RD) is an immune-mediated fibro-inflammatory condition that often causes the formation of tumefactive lesions. The discovery of IgG4-RD linked many well-known isolated conditions as a distinct multi-organ disease, and started an era of promoting investigation and treatment in relevant fields. In the thyroid gland, a subcategory of Hashimoto thyroiditis (HT) with IgG4-rich inflammation was first discovered and named IgG4 thyroiditis by our group. This subtype of HT presents with rapidly progressive clinical manifestations and destructive histopathological features underlying thyroid dysfunction, which are significantly different from the common type of HT. Moreover, other IgG4-rich thyroid conditions in patients with Graves' disease and systemic IgG4-RD have been described. These observations are most frequently reported in the Asian population for unknown reasons. Although recent studies demonstrated that IgG4 thyroiditis is a specific entity independent from IgG4-RD, recognition of this unique subset of thyroid disease has yielded important insights into understanding its pathogenesis and the development of novel therapeutic approaches.
\end{abstract}

Keywords: Thyroid; thyroiditis; autoimmune thyroid disease; immunoglobulin G4 (IgG4); Asian population

Submitted Mar 28, 2020. Accepted for publication Jun 18, 2020.

doi: $10.21037 / g s-20-417$

View this article at: http://dx.doi.org/10.21037/gs-20-417

\section{Introduction}

Immunoglobulin G4 (IgG4) is the rarest of the immunoglobulin $\mathrm{G}$ subclasses to be expressed, accounting for only $3 \%$ to $6 \%$ of all IgG in normal serum. Little attention was paid to this minor component of IgG until a unique subgroup of autoimmune pancreatitis (AIP) associated with an increased serum level of IgG4 was reported by Hamano et al. in 2001 (1). Over the next decade, similar fibro-inflammatory lesions were reported at nearly all anatomic sites. At present, these disorders are unified and recognized as a unique spectrum of inflammatory disorders, which is well-known as IgG4-related disease (IgG4-RD) (2).

In 2009, our group first described a subtype of Hashimoto thyroiditis (HT) with increased IgG4-positive plasma cells in the inflamed thyroid tissue (3), which was further demonstrated to be associated with a series of distinct clinical, serological, sonographic, and histopathological features $(4,5)$. We proposed the new terminology "IgG4 thyroiditis" to define this unique subgroup of HT with IgG4-rich inflammation. Since then, the identification of IgG4-rich inflammation in the thyroid gland has attracted growing interest. More IgG4-rich thyroid conditions have been reported recently. This expanding spectrum includes a subgroup of Graves' disease (GD), Riedel's thyroiditis (RT), and "IgG4-related thyroiditis" found in patients with IgG4$\mathrm{RD}$ in other organs (6-9).

In this review article, we first provide an overview of the general features of IgG4-RD and the most recent advances in the development of new classification criteria 
for this disease. The central focus of this review is the IgG4 thyroiditis in HT. We will introduce the history of the discovery of IgG4 thyroiditis. In particular, the research status of IgG4 thyroiditis in the Asian population is highlighted, and the relationship between IgG4 thyroiditis and systemic IgG4-RD is discussed. Other forms of IgG4rich thyroid diseases are also summarized.

\section{Overview of IgG4-RD}

IgG4-RD usually affects middle-aged to elderly individuals and has a predilection for male sex (10). The clinical signs and symptoms of patients with IgG4-RD are commonly nonspecific, and are determined by the site and numbers of affected organs and the severity of the disease. It is sometimes diagnosed unexpectedly through imaging findings or pathological examination. Diffuse or focal enlargement of the affected organs is commonly identified on imaging studies in a synchronous or metachronous pattern. Most of these radiological findings are nonspecific and mimic malignancies. Thus, accurate identification of this benign disease is essential to prevent harmful overtreatment such as cancer surgery. Cross-sectional imaging techniques, such as ultrasound, computed tomography (CT), and 2-[18F]-fluoro-2-deoxy-D-glucose positron-emission tomography/computed tomography (FDG PET/CT), help to establish a clinical diagnosis of IgG4-RD because of their ability to visualize the extent of the disease and reflect the multi-organ nature (11).

A high serum IgG4 concentration was once recognized as the hallmark and a prerequisite of IgG4-RD when its concept was initially proposed. However, it soon became apparent that serum IgG4 levels are normal in a substantial percentage of patients with IgG4-RD. Moreover, high serum IgG4 levels are observed in atopic diseases, infections, and pancreatic cancer. Even in a healthy population, up to $5 \%$ of individuals can exhibit increased IgG4 in the serum $(12,13)$. Although many experts reached a consensus that a high IgG4 level alone is no longer an independent diagnostic marker, it is still an important clue for the diagnosis of IgG4-RD when combined with the typical clinical scenario. Monitoring of the serum IgG4 concentration provides guidance in the assessment of the disease activity in some patients (2).

The definite diagnosis of IgG4-RD primarily relies on the identification of typical histological and immunohistochemical features. An international consensus statement on the pathology of IgG4-RD was published in
2012 (14). Regardless of the affected organs in this disease, there are three major histopathological characteristics associated with IgG4-RD, which include dense lymphoplasmacytic infiltrate, fibrosis arranged at least focally in a storiform pattern, and obliterative phlebitis. However, in the lung, minor salivary glands, lacrimal glands, and lymph node, storiform fibrosis and obliterative phlebitis may be absent. Other than the typical histological appearance, immunohistochemical confirmation with IgG4 should be required if possible. The number of IgG4 ${ }^{+}$ plasma cells is more sensitive and specific than the serum IgG4 titer. Quantitative analysis of IgG4, IgG, or CD138 immunostaining is recommended. $\mathrm{IgG}^{+}$plasma cells $>10 /$ $\mathrm{HPF}$ and an $\mathrm{IgG}^{+} / \mathrm{IgG}^{+}$plasma cell ratio $>40 \%$ have been used as a general threshold in the histopathological diagnosis of IgG4-RD in Japan, but other organ-specific diagnostic criteria have also been established (10).

A marked response to glucocorticoid (GC) treatment has been reported as a characteristic finding by many experts since the first description of an IgG4-related lesion in the pancreas. Currently, GCs remain the first-line therapy for patients with active, untreated IgG4-RD (15). However, many patients do not maintain their disease responses as GCs are tapered. Recently the introduction of rituximab, a select B-cell depleting agent, has brought a new impetus into the treatment of IgG4-RD. Increasing evidence has demonstrated this agent to provide effective disease control, even in steroid-refractory cases (16-18). Moreover, the use of other steroid-sparing drugs, including azathioprine, methotrexate, or mycophenolate mofetil, has been suggested for remission induction and maintenance $(15,19)$. However, currently, there is no international consensus regarding the utilization of add-on therapy with steroidsparing agents. Many questions remain to be answered in randomized, controlled clinical trials.

Although the above overview has drawn a relatively clear outline of IgG4-RD, it is still challenging to understand the epidemiology of IgG4-RD, not only because of insufficient awareness of this newly defined entity, but also due to the lack of well-established diagnostic criteria. No clinical, laboratory, radiological, or pathological findings alone provide definite evidence to accurately classify IgG4-RD. To address this issue, Japanese investigators organized by the Ministry of Health, Labour and Welfare Japan (MHLW) proposed comprehensive diagnostic criteria for IgG4-RD in 2012. It is the first joint consensus criteria integrating the clinical, serological, and histopathological evaluations of IgG4-RD. An IgG4 level $>135 \mathrm{mg} / \mathrm{dL}$ in serum, and 
IgG4 $4^{+}$plasma cell $>10 /$ high-power field (HPF), and IgG4 $4^{+}$ $\mathrm{IgG}^{+}$plasma cell ratio $>40 \%$ in tissue were used as the cutoff values. These criteria provide a useful framework for general physicians and non-specialists to identify IgG4-RD in their clinical practice (10).

The comprehensive diagnostic criteria have become well accepted mainly in Asian countries, especially in Japan since 2012. Very recently, the American College of Rheumatology (ACR) and European League Against Rheumatism (EULAR) organized an international working group including 86 multi-specialty investigators and aimed to establish international classification criteria for IgG4-RD (20). A total of 1,879 patients with either IgG4-RD or a mimicking disease were assembled by the experts from the Americas, Europe, Asia, and Australia, and used as a derivation and validation cohort, respectively. Based on a data-driven approach and multi-criterion decision analysis, a three-step classification process, including entry criteria, exclusion criteria, and inclusion criteria, was developed for IgG4RD. A potential case of IgG4-RD must meet entry criteria and not fulfill any exclusion criteria. In the next part of this process, eight weighted inclusion criteria domains, including clinical, serological, radiological, and pathological findings, must be assessed and assigned scores. A threshold of more than 20 points, which demonstrates ideal performance with high specificity (99.2\% and $97.8 \%$ ) and moderately high sensitivity $(85.5 \%$ and $82.0 \%)$ in two validation cohorts, was finally adopted. The 2019 ACR/EULAR IgG4-RD classification criteria are one of the first to include absolute exclusion criteria, which can further eliminate patients with mimicking conditions from IgG4-RD classification. The other significant change is that the 2019 ACR/EULAR classification criteria may assign a classification of IgG4$\mathrm{RD}$ even when serological and biopsy evidence is absent. This improvement will facilitate the identification of IgG4-RD in clinical practice when the serum IgG4 level remains in the normal range and or the tissue specimen is difficult to obtain from certain organs, especially the pancreas, retroperitoneum, pituitary, and ocular cavity. However, the investigators also stated that the 2019 ACR/ EULAR classification criteria are not intended for use in clinical practice but instead help to select a homogeneous population with IgG4-RD for inclusion into basic medical investigation and clinical trials (20). Until confirmed in larger clinical studies, the above criteria and scores can only support the diagnosis of IgG4-RD but cannot replace expert experience in the assessment of clinical history and cautious interpretation of examination results (21).

\section{IgG4 thyroiditis in HT}

The thyroid gland is the most common endocrine organ affected by autoimmune disease, and a high prevalence of hypothyroidism in patients with AIP was reported $(22,23)$. As the primary cause of thyroid tissue damage and hypothyroidism, HT became one of the first candidates suspected to be associated with IgG4-related conditions. In 2009, our group from Japan initially reported a small subtype of HT with substantial infiltration of $\mathrm{IgG}^{+}$ plasma cells in the thyroid tissue. Relying on IgG4 immunostaining, we proposed that $\mathrm{HT}$ can be further divided into two subcategories: IgG4 thyroiditis (IgG4 $4^{+}$ plasma cell-rich group) and non-IgG4 thyroiditis (IgG4 ${ }^{+}$ plasma cell-poor group). The former group exhibits diffuse or nodular dense infiltration of $\mathrm{IgG} 4^{+}$plasma cells, whereas the latter group has only mild to scant IgG4 $4^{+}$plasma cells. A threshold of IgG4 $4^{+}$plasma cells $>20 / \mathrm{HPF}$ and an $\mathrm{IgG}^{+} /$ $\mathrm{IgG}^{+}$plasma cell ratio $>30 \%$, which demonstrates a high specificity and sensitivity to define IgG4-rich inflammation in HT, was also proposed by our group (3).

Shortly after this study, our group performed an advanced clinical investigation based on a large cohort of HT cases retrospectively recruited from Kuma Hospital (Kobe, Japan). Using the cut-off value proposed in our previous report, 19 cases $(27 \%)$ of IgG4 thyroiditis were identified from a total of 70 patients with HT. Most importantly, a set of distinct clinical, serological, and sonographic features of IgG4 thyroiditis were discovered, and may facilitate clinical identification of this unique subgroup. First, patients in the IgG4 thyroiditis group are associated with a lower female-to-male ratio (14:5) and a younger age $(52.79 \pm 10.29$ years $)$ at presentation compared with those in the non-IgG4 thyroiditis group (48:3) (57.73 \pm 8.598 years). Second, although patients with HT generally have a chronic disease course, the IgG4 variant is associated with a shorter disease duration $(9.647 \pm 8.838$ years) before thyroidectomy than its non-IgG4 counterpart $(17.11 \pm 10.34$ years), reflecting a more rapid and aggressive course. Third, as the two main autoantibodies found in HT, the levels of both anti-thyroglobulin antibodies (Tg-Abs) and anti-thyroid peroxidase antibodies (TPO-Abs, formerly anti-microsomal antibodies) are significantly increased in IgG4 thyroiditis. Forth, patients with IgG4 thyroiditis are more likely to present with high TSH levels even when treated using levothyroxine. On ultrasound examination, the IgG4 thyroiditis group has more marked diffuse low echogenicity than the non-IgG4 thyroiditis group. 

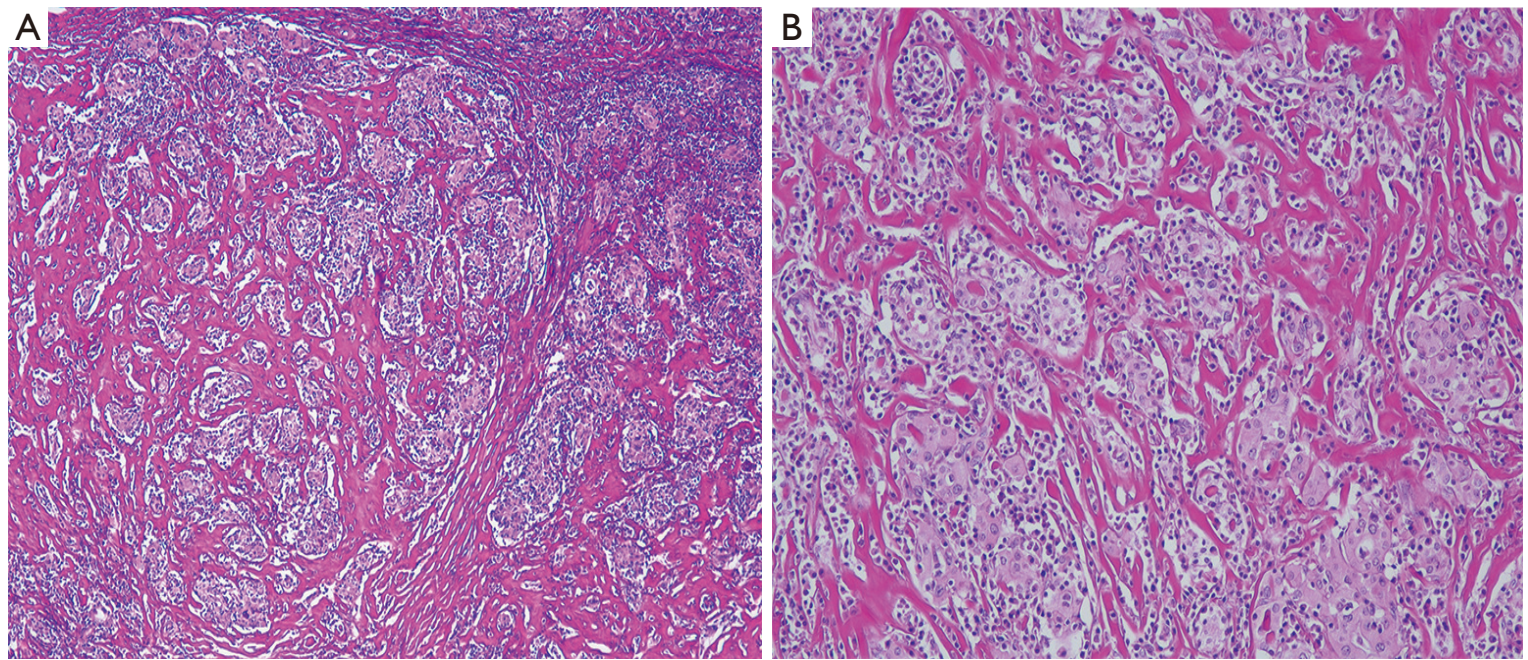

Figure 1 Histopathological features of IgG4 thyroiditis. (A) Dense stromal fibrosis, lymphoplasmacytic infiltration, and numerous microfollicles are noticed in the thyroid tissue (HE, $\times 40)$. (B) Atrophic thyroid follicles separated by the deposition of fibrous tissue with lymphoplasmacytic infiltration in the interfollicular area $(\mathrm{HE}, \times 200)$.
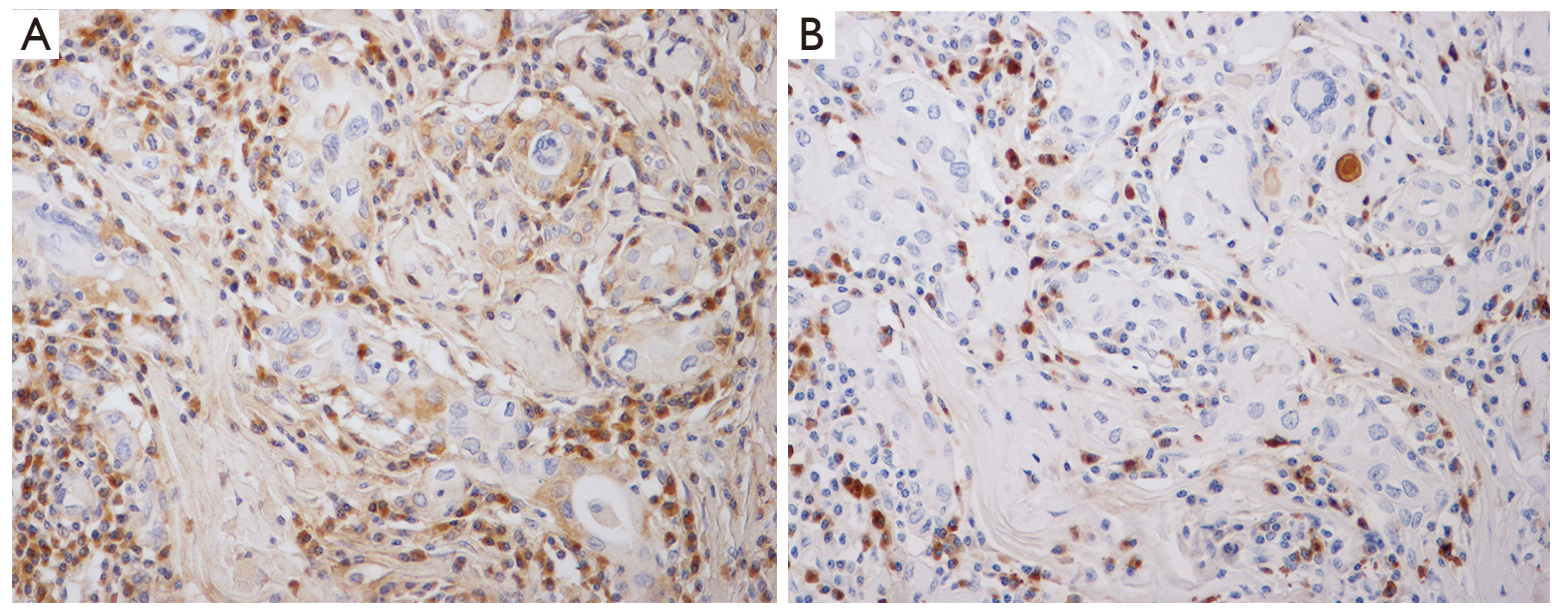

Figure 2 A marked increase in $\mathrm{IgG}^{+}(\mathrm{A})$ and $\operatorname{IgG}^{+}(\mathrm{B})$ plasma cells among the inflammatory infiltrates showing a high $\mathrm{IgG}^{+} / \mathrm{IgG}{ }^{+}$ratio $(\operatorname{IgG}$ and IgG4; IHC, $\times 400)$.

Furthermore, although only a small number of patients had serum samples available for retrospective serum IgG subclasses analysis, a significant increase in the serum IgG4 level (range, $167-459 \mathrm{mg} / \mathrm{dL}$; reference value of serum IgG4: 4.8-105 mg/dL), which demonstrated a marked reduction after surgery (range, $25.6-97.8 \mathrm{mg} / \mathrm{dL}$ ), was identified in a subset of patients with IgG4 thyroiditis $(4,24)$.

In clinical practice, histopathological evidence is not required by endocrinologists to diagnose HT because, in most instances, it is a medically treated disease. However, pathological assessment and the subsequent immunostaining examination are the keys to assign a subclassification of IgG4 thyroiditis. A series of typical pathological features of IgG4 thyroiditis was discovered by our group in 2009 and later refined in 2012. Histologically, the IgG4 thyroiditis group exhibits characteristic lymphoplasmacytic infiltration, dense stromal fibrosis, marked follicular cell degeneration, numerous microfollicles, and notable giant cell/histiocyte infiltration (5) (Figure 1). At the same time, these features are either absent or not noticeable in the non-IgG4 group. 
Table 1 Reported IgG4 thyroiditis in Asian population

\begin{tabular}{|c|c|c|c|c|}
\hline Source & Country & Database & Design & Prevalence \\
\hline Li et al., 2010 (4) & Japan & Surgical pathology & Retrospective & $19 / 70$ \\
\hline Li et al., 2012 (5) & Japan & Surgical pathology & Retrospective & $28 / 105$ \\
\hline Zhang et al., 2014 (27) & China & Surgical pathology & Retrospective & $12 / 53$ \\
\hline Takeshima et al., 2015 (28) & Japan & Serologic & Prospective & $6 / 149$ \\
\hline Zhao et al., 2018 (29) & Singapore & Surgical pathology & Case report & - \\
\hline
\end{tabular}

The fibroinflammatory changes and increased $\mathrm{IgG} 4^{+}$plasma cell infiltration in the thyroid tissue are highly similar to those in other organs affected in IgG4-RD (Figure 2). Together with the frequent combination with AIP, we initially hypothesized a close link between IgG4 thyroiditis and IgG4-RD (25).

\section{IgG4 thyroiditis in the Asian population}

Since the first report of IgG4 thyroiditis, endocrinologists and pathologists started investigating this field, and the idea of IgG4 thyroiditis as a unique subcategory of HT has been confirmed. In 2014, Brito-Zerón et al. summarized the geographic origin of 3,543 reported cases of IgG4-RD, $2,621(74 \%)$ of which were from Asia (26). Up to now, a substantial number of reports focusing on IgG4 thyroiditis or IgG4-RD was mainly from Asian countries, where the entity was initially described (Table 1).

In 2014, Zhang et al. from China reported 12 out of 53 cases $(22.6 \%)$ of surgically treated HT with increased $\mathrm{IgG}^{+}$plasma cell infiltration at the tissue level. The IgG4 thyroiditis patients identified in their cohort were also younger and had marked stromal fibrosis. Although no significant differences were found in the levels of serum IgG4, they found that the level of IgG4 binding to specific thyroid antigens, such as Tg and TPO, was significantly higher in the IgG4 thyroiditis group (27). In the same year, Kawashima et al. from Japan performed a serological study to screen total IgG levels in patients with HT. Twentyfour of 94 patients (25.5\%) had high serum IgG levels. Five of the 24 patients had an increased IgG4 concentration (>135 $\mathrm{mg} / \mathrm{dL}$ ). Among these 5 , thyroid biopsy was performed on one patient with diffuse lymphoplasmacytic infiltration, severe stromal fibrosis, lymphoid follicle formation, and numerous $\operatorname{IgG} 4^{+}$plasma cells replacing thyroid parenchyma, which were consistent with the typical histological features of IgG4 thyroiditis (22). Later in 2015, Takeshima et al. from Japan prospectively recruited 149 patients with HT, and aimed to clarify the distribution of serum IgG4 and its association with clinical features in HT. In their report, six patients (4\%) had a high serum IgG4 level above $135 \mathrm{mg} / \mathrm{dL}$, which was found to be related to large hypo-echoic areas on ultrasound examination. Two of the six patients exhibited swelling of bilateral lacrimal glands and pituitary glands, which was considered to be associated with extrathyroid IgG4 lesions (28).

Apart from the above studies performed on relatively large numbers of patients with HT, some individual case reports also provide useful information for us to confirm the existence of IgG4 thyroiditis, and to understand its natural history and clinicopathological features. In 2018, Zhao et al. from Singapore reported the first case of IgG4 thyroiditis in the Malay population in Southeast Asia. A 47-yearold woman presented with progressively painless neck swelling over three years. CT revealed homogenous and diffuse thyroid enlargement (up to $13 \mathrm{~cm}$ ) with retrosternal extension. The thyroid function test demonstrated subclinical hypothyroidism. She underwent emergency total thyroidectomy due to extrinsic upper airway compression by the thyroid mass, which caused hypercapnic respiratory failure. Histological examination revealed dense lymphoplasmacytic infiltration, fibrosis, and increased IgG4 $4^{+}$plasma cells ( $>40 / \mathrm{HPF}$ and $\mathrm{IgG} 4^{+} / \mathrm{IgG}^{+}$ratio $>50 \%$ ), in addition to other characteristics of HT. No significantly increased fludeoxyglucose uptake in other areas after surgery was noted on PET/CT. Although such a rapidly progressive case of HT is less common in clinical practice, physicians should keep in mind that this manifestation may be related to the unique nature of IgG4-rich inflammation and emergency total thyroidectomy should be considered if 
necessary (29).

Although recently Jokish et al. from Germany and Raess et al. from the United States identified an IgG4-rich group in their cohorts by investigating surgical specimens of HT, a lower rate of IgG4 thyroiditis (12\%) (30) and absence of a significant association between increased $\mathrm{IgG} 4^{+}$plasma cells in thyroid tissue and the degree of stromal fibrosis were respectively reported by the authors (31). The discrepancies between Asian and Western studies on IgG4 thyroiditis may be explained by genetic, dietary, or geographic differences. For example, the German group investigated Caucasian patients with HT mainly from an iodine-deficient alpine area of Southern Germany and Austria, where the incidence of HT is lower than in East Asia. On the other hand, IgG4 thyroiditis may be significantly underdiagnosed, especially in Europe and North America, where the awareness of its existence may be lower than in Asian countries. Therefore, further international and multidisciplinary studies on IgG4 thyroiditis are necessary to broaden our knowledge of this unique subcategory globally.

\section{Other IgG4-rich thyroid diseases}

In addition to the IgG4 thyroiditis in $\mathrm{HT}$, other IgG4-rich inflammatory lesions have been reported in recent years. Graves' disease (GD), another common autoimmune thyroid disorder that shares many underlying tenets with HT, has also been linked to the $\mathrm{IgG}^{+}$plasma cell-rich condition in recent years. Of note, similar to IgG4 thyroiditis in HT, most of the studies this topic was reported from Asia.

In 2013, Nishihara et al. from Japan reported a 47-yearold woman with GD who underwent methimazole (MMI) therapy to maintain a euthyroid state, but eventually developed overt hypothyroidism and diffuse enlargement of the thyroid gland six months later after the withdrawal of MMI. Histological examination revealed fibroinflammatory lesions with increased $\mathrm{IgG}^{+}$plasma cells $\left(\mathrm{IgG}^{+}\right.$plasma cell $>200 / \mathrm{HPF}$ and $\mathrm{IgG} 4^{+} / \mathrm{IgG}^{+}$plasma cell ratio $>40 \%$ ). Serological IgG4 levels were high before thyroidectomy and decreased to within the normal range three months after surgery (6). Indeed, this new report raised great interest for endocrinologists to investigate the relationship between GD and IgG4-rich inflammation. In 2014, Takeshima et al. from Japan performed a prospective study by analyzing serum IgG4 levels in 109 patients with GD. Seven of 109 patients $(6.4 \%)$ had increased circulating IgG4 levels. They were older (in contrast to HT) at diagnosis, had increased hypoechoic areas in the thyroid, and responded excellently to anti-thyroid drug treatment (7). This study highlighted the existence of IgG4-rich GD and its potential clinical significance. As one of the three core clinical characteristics of GD, Graves' ophthalmopathy (GO) affecting $25-50 \%$ of GD patients, was also confirmed to be related to the IgG4 level. Yu et al. from Korea conducted a prospective casecontrol study composed of 64 GD patients and 64 sex- and age-matched euthyroid subjects. They found that serum IgG4 levels were high $(>86.4 \mathrm{mg} / \mathrm{dL})$ in 6 of 64 patients $(9.4 \%)$ of the GD group, whereas all subjects in the control group had normal IgG4 levels. They also suggested that the serum IgG4 level was an independent factor associated with the development and grade of GO in patients with GD. IgG4 levels increased in parallel with the severity and clinical activity of GO (32). However, the relationship between GD and IgG4-rich inflammation needs to be further validated in larger patient cohorts and Western populations.

In addition to GD, Watanabe et al. analyzed 114 patients with IgG4-RD and examined potentially related thyroid lesions. They found 22 patients $(19 \%)$ revealing elevated serum thyroid stimulating hormone ( $>4 \mathrm{mIU} / \mathrm{L})$, either overt (FT4: $<1 \mathrm{ng} / \mathrm{dL}$ ) or subclinical (FT4: $>1 \mathrm{ng} / \mathrm{dL}$ ) hypothyroidism. The serum concentration of IgG4 was significantly higher in these patients than in those with euthyroidism. In contrast to IgG4-rich inflammation in HT and GD, diffusely enlarged thyroid goiter and thyroid autoantibodies were uncommon. Although increased IgG4 plasma cell infiltration was subsequently confirmed at the tissue level in only one patient, the authors proposed this type of thyroid disease to be "IgG4-related thyroiditis" (9).

The above thyroid lesions were previously regarded as isolated entities. However, they overlap with each other and the destructive inflammation can explain this overlap with increased IgG4 $4^{+}$plasma cells, which leads to hypothyroidism regardless of the differing etiology. Regarding the nomenclature of IgG4-rich thyroid inflammation, we propose IgG4 thyroiditis because this simple descriptive term can cover these different thyroid diseases as a unified concept without speculation on pathogenesis. The terms "IgG4-related thyroiditis" and "IgG4-related thyroid disease" should be used only when systemic IgG4-RD is present.

\section{IgG4 thyroiditis and systemic IgG4-RD}

$\mathrm{RT}$ is a sporadic disease with an incidence of $0.06 \%$ according to the Mayo Clinic (33). In a national investigation 
of RT from Japan, only ten patients were diagnosed pathologically over 25 years $(33,34)$. Although only one previously reported case of RT fulfilled the comprehensive diagnostic criteria, RT has been firmly considered a member of IgG4-RD by some investigators (8). On the other hand, the relationship between IgG4 thyroiditis in HT and IgG4-RD has become controversial in recent years. Although diffuse enlargement of the thyroid gland, male predisposition, fibroinflammatory change, and increased IgG4 levels both in tissue and serum in IgG4 thyroiditis represent a similar phenotype to that of IgG4-RD, there are no reports on extrathyroid involvement in IgG4 thyroiditis $(5,27,30)$. Histologically, storiform fibrosis and obliterative phlebitis have been recognized as major pathological features of IgG4-RD in other organs (14). However, typical storiform fibrosis is rare and no evidence of obliterative phlebitis was identified in the IgG4 thyroiditis cases in previous reports. Although no single case of HT or RT was involved in the development and validation processes of the 2019 ACR/EULAR classification criteria for IgG4$\mathrm{RD}, \mathrm{RT}$ was proposed as the only thyroid manifestation of IgG4-RD, and HT was listed in the exclusion criteria by this new classification criteria. The authors missed a "not" when they stated that "Patients with IgG4-RD can certainly have HT separately from IgG4-RD, but HT is part of the IgG4-RD spectrum" in the last sentence of "specific disease exclusions". Recently, Inomata et al. from our group reported that the major auto-antigen recognized by serum IgG4 antibodies in patients with IgG4 thyroiditis is thyroglobulin and its isoforms (35). However, other antigens, such as Annexin A11, galectin-3, and laminin 511, which are expressed in almost all organs, were reported to be the main targets of IgG4 antibodies in systemic IgG4-RD (36-38). These observations may explain the solitary nature of IgG4 thyroiditis and its difference from systemic IgG4$\mathrm{RD}$, and stimulate further studies towards the in-depth understanding of the pathogenesis of IgG4 thyroiditis.

\section{Clinical significance of IgG4 thyroiditis and the discovery of borderline lesions}

HT is one of the most common autoimmune diseases in humans responsible for considerable morbidity, approximately $0.5 \%$, in the general population. Although IgG4 thyroiditis is a minor subcategory of HT, with an incidence of approximately $4 \%$ in the general HT population and $12.6-34.8 \%$ in surgically treated cases (39), the discovery of this subgroup will affect a large number of patients with HT worldwide and prompt further investigation of potential alternative therapeutic options for patients in the future.

Compared with the common type of HT, IgG4 thyroiditis usually demonstrates destructive fibroinflammatory change, which results in the loss of normal thyroid structure and function, leading to hypothyroidism. A rapidly progressive clinical course of IgG4 thyroiditis make the differential diagnosis from malignant tumor more difficult and may suggest a less favorable prognosis. Very recently, our group confirmed that the cut-off value of thyroid-specific diagnostic criteria (CVT: IgG4 ${ }^{+}$plasma cells $>20 / \mathrm{HPF}$, and $\mathrm{IgG}^{+} / \mathrm{IgG}^{+}$plasma cell ratio $>30 \%$ ) can precisely define a significant IgG4 $4^{+}$plasma cell count in IgG4 thyroiditis. More importantly, the thyroid-specific cut-off value can more efficiently identify borderline cases, which may represent an "early-phase" of IgG4 thyroiditis (40). Therefore, these discoveries will facilitate the early detection and intervention for IgG4 thyroiditis in clinical and pathological practice in the near future.

\section{Conclusions}

An improved understanding of systemic IgG4-RD has led to the initial discovery of IgG4 thyroiditis. Within one decade, these two new disease entities have become characterized by IgG4-rich inflammation. Following continued clinical observation and basic investigation, IgG4 thyroiditis is now recognized as a unique subcategory of autoimmune thyroid disease and an independent organ-specific condition different from systemic IgG4-RD. The development of precise diagnostic criteria for IgG4 thyroiditis and improved understanding of the related research status and prospects in the Asian population may improve therapeutic approaches and the quality of life for patients with $\operatorname{IgG} 4$ thyroiditis in Asia.

\section{Acknowledgments}

Funding: This work was supported by the National Natural Science Foundation of China No. 81300647 and a Startup Foundation for Young Scientists from Shandong First Medical University to Yaqiong Li.

\section{Footnote}

Provenance and Peer Review: This article was commissioned by the Guest Editor (Kennichi Kakudo) for the series "Asian 
and Western Practice in Thyroid Pathology: Similarities and Differences" published in Gland Surgery. The article was sent for external peer review organized by the Guest Editor and the editorial office.

Peer Review File: Available at http://dx.doi.org/10.21037/gs20-417

Conflicts of Interest: All authors have completed the ICMJE uniform disclosure form (available at http://dx.doi. org/10.21037/gs-20-417). The series "Asian and Western Practice in Thyroid Pathology: Similarities and Differences" was commissioned by the editorial office without any funding or sponsorship. KK serves as the unpaid Guest Editor of the series and an unpaid editorial board member of Gland Surgery from Sep 2018 to Aug 2020. The authors have no other conflicts of interest to declare.

Ethical Statement: The authors are accountable for all aspects of the work in ensuring that questions related to the accuracy or integrity of any part of the work are appropriately investigated and resolved.

Open Access Statement: This is an Open Access article distributed in accordance with the Creative Commons Attribution-NonCommercial-NoDerivs 4.0 International License (CC BY-NC-ND 4.0), which permits the noncommercial replication and distribution of the article with the strict proviso that no changes or edits are made and the original work is properly cited (including links to both the formal publication through the relevant DOI and the license). See: https://creativecommons.org/licenses/by-nc-nd/4.0/.

\section{References}

1. Hamano H, Kawa S, Horiuchi A, et al. High serum IgG4 concentrations in patients with sclerosing pancreatitis. $\mathrm{N}$ Engl J Med 2001;344:732-8.

2. Kamisawa T, Zen Y, Pillai S, et al. IgG4-related disease. Lancet 2015;385:1460-71.

3. Li Y, Bai Y, Liu Z, et al. Immunohistochemistry of IgG4 can help subclassify Hashimoto's autoimmune thyroiditis. Pathol Int 2009;59:636-41.

4. Li Y, Nishihara E, Hirokawa M, et al. Distinct clinical, serological, and sonographic characteristics of hashimoto's thyroiditis based with and without IgG4-positive plasma cells. J Clin Endocrinol Metab 2010;95:1309-17.

5. Li Y, Zhou G, Ozaki T, et al. Distinct histopathological features of Hashimoto's thyroiditis with respect to IgG4related disease. Mod Pathol 2012;25:1086-97.

6. Nishihara E, Hirokawa M, Takamura Y, et al. Immunoglobulin G4 thyroiditis in a Graves' disease patient with a large goiter developing hypothyroidism. Thyroid 2013;23:1496-7.

7. Takeshima K, Inaba H, Furukawa $Y$, et al. Elevated serum immunoglobulin G4 levels in patients with Graves' disease and their clinical implications. Thyroid 2014;24:736-43.

8. Dahlgren M, Khosroshahi A, Nielsen GP, et al. Riedel's thyroiditis and multifocal fibrosclerosis are part of the IgG4-related systemic disease spectrum. Arthritis Care Res (Hoboken) 2010.

9. Watanabe T, Maruyama M, Ito T, et al. Clinical features of a new disease concept, IgG4-related thyroiditis. Scand J Rheumatol 2013;42:325-30.

10. Umehara H, Okazaki K, Masaki Y, et al. Comprehensive diagnostic criteria for IgG4-related disease (IgG4-RD), 2011. Mod Rheumatol 2012;22:21-30.

11. Katabathina VS, Khalil S, Shin S, et al. Immunoglobulin G4-Related Disease: Recent Advances in Pathogenesis and Imaging Findings. Radiol Clin North Am 2016;54:535-51.

12. Ryu JH, Horie R, Sekiguchi H, et al. Spectrum of Disorders Associated with Elevated Serum IgG4 Levels Encountered in Clinical Practice. Int J Rheumatol 2012;2012:232960.

13. Sah RP, Chari ST. Serologic issues in IgG4-related systemic disease and autoimmune pancreatitis. Curr Opin Rheumatol 2011;23:108-13.

14. Deshpande V, Zen Y, Chan JK, et al. Consensus statement on the pathology of IgG4-related disease. Mod Pathol 2012;25:1181-92.

15. Khosroshahi A, Wallace ZS, Crowe JL, et al. International Consensus Guidance Statement on the Management and Treatment of IgG4-Related Disease. Arthritis Rheumatol 2015;67:1688-99.

16. Khosroshahi A, Carruthers MN, Deshpande V, et al. Rituximab for the treatment of IgG4-related disease: lessons from 10 consecutive patients. Medicine (Baltimore) 2012;91:57-66.

17. Hart PA, Topazian MD, Witzig TE, et al. Treatment of relapsing autoimmune pancreatitis with immunomodulators and rituximab: the Mayo Clinic experience. Gut 2013;62:1607-15.

18. Carruthers MN, Topazian MD, Khosroshahi A, et al. Rituximab for IgG4-related disease: a prospective, openlabel trial. Ann Rheum Dis 2015;74:1171-7.

19. Raina A, Yadav D, Krasinskas AM, et al. Evaluation and 
Management of Autoimmune Pancreatitis: Experience at a Large US Center. Am J Gastroenterol 2009.

20. Wallace ZS, Naden RP, Chari S, et al. The 2019 American College of Rheumatology/European League Against Rheumatism classification criteria for IgG4-related disease. Ann Rheum Dis 2020;79:77-87.

21. Lang D, Zwerina J, Pieringer H. IgG4-related disease: current challenges and future prospects. Ther Clin Risk Manag 2016;12:189-99.

22. Kawashima ST, Tagami T, Nakao K, et al. Serum levels of $\mathrm{IgG}$ and IgG4 in Hashimoto thyroiditis. Endocrine 2014;45:236-43.

23. Komatsu K, Hamano H, Ochi Y, et al. High prevalence of hypothyroidism in patients with autoimmune pancreatitis. Dig Dis Sci 2005;50:1052-7.

24. Kakudo K, Li Y, Taniguchi E, et al. IgG4-related disease of the thyroid glands. Endocr J 2012;59:273-81.

25. Li Y, Nishihara E, Kakudo K. Hashimoto's thyroiditis: old concepts and new insights. Curr Opin Rheumatol 2011;23:102-7.

26. Brito-Zeron P, Ramos-Casals M, Bosch X, et al. The clinical spectrum of IgG4-related disease. Autoimmun Rev 2014;13:1203-10.

27. Zhang J, Zhao L, Gao Y, et al. A classification of Hashimoto's thyroiditis based on immunohistochemistry for IgG4 and IgG. Thyroid 2014;24:364-70.

28. Takeshima K, Ariyasu H, Inaba H, et al. Distribution of serum immunoglobulin G4 levels in Hashimoto's thyroiditis and clinical features of Hashimoto's thyroiditis with elevated serum immunoglobulin G4 levels. Endocr J 2015;62:711-7.

29. Zhao Z, Lee YJ, Zheng S, et al. IgG4-Related Disease of the Thyroid Gland Requiring Emergent Total Thyroidectomy: A Case Report. Head Neck Pathol 2019;13:523-7.

30. Jokisch F, Kleinlein I, Haller B, et al. A small subgroup of Hashimoto's thyroiditis is associated with IgG4-related

Cite this article as: Li Y, Inomata K, Nishihara E, Kakudo K. IgG4 thyroiditis in the Asian population. Gland Surg 2020;9(5):1838-1846. doi: 10.21037/gs-20-417 disease. Virchows Arch 2016;468:321-7.

31. Raess PW, Habashi A, El Rassi E, et al. Overlapping Morphologic and Immunohistochemical Features of Hashimoto Thyroiditis and IgG4-Related Thyroid Disease. Endocr Pathol 2015;26:170-7.

32. Yu SH, Kang JG, Kim CS, et al. Clinical Implications of Immunoglobulin G4 to Graves' Ophthalmopathy. Thyroid 2017;27:1185-93.

33. Fatourechi MM, Hay ID, McIver B, et al. Invasive fibrous thyroiditis (Riedel thyroiditis): the Mayo Clinic experience, 1976-2008. Thyroid 2011;21:765-72.

34. Takeshima K, Inaba H, Ariyasu H, et al. Clinicopathological features of Riedel's thyroiditis associated with IgG4-related disease in Japan. Endocr J 2015;62:725-31.

35. Inomata K, Kurisaki H, Yamashita H, et al. Identification of Thyroglobulin and its isoforms as Target Antigens for IgG4 thyroiditis. J Clin Cell Immunol 2018;9:568.

36. Hubers LM, Vos H, Schuurman AR, et al. Annexin A11 is targeted by IgG4 and IgG1 autoantibodies in IgG4-related disease. Gut 2018;67:728-35.

37. Shiokawa M, Kodama Y, Sekiguchi K, et al. Laminin 511 is a target antigen in autoimmune pancreatitis. Sci Transl Med 2018;10.

38. Perugino CA, AlSalem SB, Mattoo H, et al. Identification of galectin-3 as an autoantigen in patients with IgG4-related disease. J Allergy Clin Immunol 2019;143:736-45.e6.

39. Rotondi M, Carbone A, Coperchini F, et al. DIAGNOSIS OF ENDOCRINE DISEASE: IgG4related thyroid autoimmune disease. Eur J Endocrinol 2019;180:R175-R83.

40. Li Y, Wang X, Liu Z, et al. Hashimoto's Thyroiditis with Increased IgG4-Positive Plasma Cells: Using ThyroidSpecific Diagnostic Criteria May Identify Early Phase IgG4 Thyroiditis. Thyroid 2020;30:251-61. 